\title{
EDITORIAL
}

\section{Las políticas de acceso abierto y su impacto en la ciencia latinoamericana}

José F. González-Maya - Sociedad Colombiana de Mastozoología, Director Mammalogy Notes. Centro del Cambio Global y la Sustentabilidad en el Sureste AC \& Instituto de Ecología, UNAM. ProCAT Colombia \& The Sierra to Sea Institute. Bogotá, Colombia. jfgonzalezmaya@gmail.com.

Diego A. Gómez-Hoyos - The Sierra to Sea Institute, Puntarenas, Costa Rica \& Grupo de Investigación y Asesoría en Estadística, Universidad del Quindío, Armenia, Colombia. dagomezh@uqvirtual.edu.co

María del Pilar Sáenz - Fundación Karisma, Bogotá, Colombia. mpsaenz@karisma.org.co

El crecimiento y desarrollo científico son considerados uno de los motores y promotores de cambio en la sociedad y una de las bases necesarias para el desarrollo de un país (González-Maya \& Cepeda 2011, Blanco 2013). Tanto el crecimiento como el desarrollo están determinados por dos factores preponderantes: en primer lugar la inversión que realiza cada país en su desarrollo científico y tecnológico, y segundo, en el acceso, apropiación y uso que tiene la academia, la sociedad y los mismos tomadores de decisiones sobre el conocimiento generado (González-Maya et al. 2010). Estos dos procesos son probablemente los determinantes fundamentales del impacto que puede tener potencialmente la ciencia y la tecnología en el país, y determinarán de forma necesaria el papel que jugará en su desarrollo y sus políticas.

Latinoamérica ha estado históricamente rezagada del desarrollo científico global, y sólo hasta ahora ha sido más evidente y claro el crecimiento de la ciencia latinoamericana, en especial para algunos países de la región como México, Argentina y Brasil. La inversión en ciencia y tecnología en Latinoamérica, como indicador macroeconómico de su desarrollo, se encuentra significativamente por debajo de los países más desarrollados; mientras países como Estados Unidos, Canadá o Finlandia invierten el 2,76, 1,78 y 3,79 \% del Producto Interno Bruto (según datos del Banco Mundial a 2011), el promedio latinoamericano alcanza tan sólo el 0,82 \%, siendo de nuevo resaltables el 1,21 de Brasil, el 0,64 de Argentina y el 0.43 y $0.42 \%$ de Uruguay y México, respectivamente. Como es claro, estos porcentajes son significativamente diferentes entre el llamado "primer mundo" y Latinoamérica, lo que de entrada marca una diferencia fundamental en el desarrollo y papel potencial de la ciencia en el desarrollo de nuestros países. Sin embargo, no sólo por esta razón la ciencia en nuestros países no alcanza su papel de motor del desarrollo. La información generada en el país generalmente no es accesible por la mayoría de la población, incluyendo la misma academia y por supuesto no alcanza a tomadores de decisiones o a la población en general. Existen razones subyacentes que afectan esta problemática, incluyendo el divorcio entre ciencia y política, dónde la información nunca llega a ser traducida al lenguaje político o común (González-Maya \& Cepeda 2011) y adicionalmente, lo relacionado con la carencia y falta de medios para acceder a esta información.

Tradicionalmente, los estándares y paradigmas científicos en Latinoamérica se han apegado y han seguido de cerca aquellos impuestos o establecidos por el sistema estadounidense o europeo de generación y difusión de la

Mammalogy Notes | Notas Mastozoológicas

Sociedad Colombiana de Mastozoología

Vol. 1 Num. 2| 2014 
ciencia. Este paradigma ha generado una de las industrias más lucrativas del planeta, la que involucra la publicación y venta de la información científica por medio de las grandes compañías de publicación. El "mainstream" o ciencia de alta calidad e impacto es publicada en su mayoría en revistas que pertenecen o son manejadas por estas compañías, y su acceso representa una inversión económica significativa (en algunos casos alcanza hasta 1 millón de dólares americanos) para instituciones, y ni hablar de investigadores o estudiantes independientes. En promedio, el cobro por acceder a un artículo en una revista de alto impacto ronda los 30 dólares americanos, lo que representa una limitante singular para "construir ciencia", pues en promedio entre 30 y 40 citaciones de una publicación de alta calidad representarían alrededor de 900-1200 dólares, en la mayoría de casos impagables por un investigador latinoamericano. De hecho, la Declaración sobre la ciencia y el uso del saber científico (Budapest, Hungría 1999) indica que "la mayor parte de los beneficios derivados de la ciencia están desigualmente distribuidos a causa de las asimetrías estructurales existentes entre los países, las regiones y los grupos sociales, así como entre los sexos. Conforme el saber científico se ha transformado en un factor decisivo de la producción de riquezas, su distribución se ha vuelto más desigual.”

Muchas instituciones en la región han logrado cubrir sus suscripciones a las principales "redes de conocimiento", sin embargo, no es la generalidad y así numerosos académicos, estudiantes, profesores e investigadores no tienen acceso legal al conocimiento generado a escala global. Esto sumado a que la mayoría no es en su idioma, pero además, que representa una discusión ética de grandes proporciones que viene siendo más y más común entre la comunidad científica: ¿si el dinero de los países y sus contribuyentes ya pagaron por una investigación, porque debe pagar por acceder a sus resultados? No es el interés de esta nota editorial entrar a esa discusión pero sí resaltar la necesidad de un nuevo enfoque en la ciencia latinoamericana. Uno posible es apostar por el acceso abierto.

La literatura referida al acceso abierto es digital, en línea, sin cargo y está libre de la mayoría de las restricciones en términos de derechos de autor y licencias (Suber, 2012). Existen tres definiciones formales sobre acceso abierto: Budapest (BOAI, 2002), Bethesda (BSOAP, 2003) y Berlin (BDOA, 2003). Las tres definiciones coinciden en que eliminar las barreras de precios no es suficiente aun cuando esas barreras de precios constituyen la parte principal del problema para el cual el acceso abierto es la solución. No hay un acuerdo sobre cuáles de las otras barreras, relacionadas con los usos y reusos de las obras, son fundamentales. De hecho las tres definiciones se diferencian en la flexibilidades que aceptan para el uso y reuso de las obras, lo que ha generado en la práctica diferentes tipos de implementaciones: algunos proveedores de acceso abierto permiten la reutilización comercial, otros permiten realizar trabajos derivados, otros se reservan el acceso por cortos periodos de tiempo antes de liberar las obras (embargo).

La base legal del acceso abierto es el consentimiento del poseedor de los derechos de autor, por lo que para su adopción no requiere la abolición, reforma o violación de la ley de derecho de autor. Tampoco requiere que los poseedores del derecho de autor renuncien a todos los derechos sino que expresen cuales derechos se reservan. Este nuevo modelo de algunos derechos reservados, se manifiesta a través del uso de licencias. Las licencias aceptadas como estándar de facto internacional para el acceso abierto son las Creative Commons, sin embargo los poseedores del derecho de autor pueden crear sus propias licencias o declaraciones de autorización, y anexarlas a sus trabajos o utilizar otros tipos de licenciamiento.

Si bien es posible utilizar este mismo enfoque para otras obras, incluso fuera del ámbito científico y tecnológico, muchas iniciativas de acceso abierto se centran en garantizar el acceso como mínimo a la investigación financiada por fondos públicos.

El acceso abierto sirve a los intereses de muchos grupos: 
- Autores: el acceso abierto amplía su audiencia y aumenta la visibilidad e impacto de su trabajo.

- Lectores: el acceso abierto proporciona acceso libre de barreras a la literatura que necesitan para la investigación. Incrementa su comodidad, alcance y poder de recuperación de la información. También brinda acceso libre de barreras al software que asiste a los lectores en sus búsquedas. La literatura gratuita en línea consta de datos gratuitos en línea para software que facilita la búsqueda de textos completos, indexación, extracción, elaboración de resúmenes, traducción, consulta, conexión, envío de alertas y otras formas de procesamiento y análisis.

- Docentes y estudiantes: el acceso abierto pone a ricos y pobres en igualdad de condiciones en lo que respecta a estos recursos clave, y elimina la necesidad de permisos para reproducir y distribuir contenido.

Dadas las limitantes antes mencionadas y las ventajas manifiestas aún nos podemos preguntar, ¿por qué el crecimiento exponencial de opciones de acceso abierto a nivel global y regional? La respuesta es clara, el acceso abierto asegura la lectura y uso de la información, facilita los procesos de construcción científica y sobretodo, aboga por la equidad en el acceso a la información, la mayoría de veces financiado con fondos públicos.

\section{Referencias}

GonzÁlez-Maya, J. F. AND A. A. CePedA. 2011. Editorial: Ciencia y publicación en Latinoamérica: retos, perspectivas y pautas. Revista Mexicana de Mastozoología 15:4-7.

GONZÁlEZ-MAYA, J. F., et al. 2010. Revistas científicas para la conservación en Latinoamérica : una necesidad, un compromiso y una oportunidad para la región. Revista Latinoamericana de Conservación $1: 5-8$.

BLANCO RANGEL, I. 2013. La apropiación social de la ciencia, la tecnología y la innovación, y los organismos de cooperación internacional. Ágora U.S.B 13:179-189.

SUBER, Peter. 2012 Open Access. The MIT Press Essential Knowledge Series: p4-ss

Budapest Open Access Iniciative. 2002.

http://www.budapestopenaccessinitiative.org/translations/spanish-translation consultado 09/10/2014

Bethesda Statement on Open Access Publishing. 2003. http://ictlogy.net/articles/bethesda_es.html consultado 09/10/2014

Berlin Declaration on Open Access to Knowledge in the Sciences and Humanities, 2003 http://openaccess.mpg.de/Berlin-Declaration consultado 09/10/2014 